\title{
Mechanically driven grain boundary relaxation: a mechanism for cyclic hardening in nanocrystalline $\mathrm{Ni}$
}

\author{
Timothy J. Rupert and Christopher A. Schuh* \\ Department of Materials Science and Engineering, Massachusetts Institute of Technology, \\ 77 Massachusetts Avenue, Cambridge, MA 02139, USA
}

(Received 27 June 2011; final version received 28 August 2011)

\begin{abstract}
Molecular dynamics simulations are used to show that cyclic mechanical loading can relax the non-equilibrium grain boundary (GB) structures of nanocrystalline metals by dissipating energy and reducing the average atomic energy of the system, leading to higher strengths. The GB processes that dominate deformation in these materials allow low-energy boundary configurations to be found through kinematically irreversible structural changes during cycling, which increases the subsequent resistance to plastic deformation.
\end{abstract}

Keywords: nanocrystalline materials; mechanical properties; grain boundary relaxation; molecular dynamics; plastic deformation

\section{Introduction}

The high strength [1,2], wear resistance [3-5], and fatigue resistance [6,7] of nanocrystalline metals are of significant scientific and technological interest, but have been primarily studied with a focus on the impact of grain size $(d)$. The suppression of traditional dislocation plasticity mechanisms and the emergence of grain boundary (GB) dominated deformation mechanisms, along with the associated shifts in behavior such as the Hall-Petch breakdown, are now well documented as a function of grain size [8]. Because of the inflated importance of GBs in nanocrystalline materials, however, not only the grain size but also the GB structural state can dramatically influence the mechanical response of nanocrystalline materials. Lowtemperature annealing experiments, which leave grain size unchanged but eliminate excess GB defects [9], can lead to significant increases in the hardness and yield strength of nanocrystalline metals $[10,11]$.

Because evolution of the atomic GB state is a subtle structural change and is difficult to track with experimental methods, molecular dynamics (MD) simulations are a useful tool for investigating GB relaxation and its effect on mechanical behavior. Vo et al. $[12,13]$ connected relaxation strengthening to a reduction in the number of GB atoms during thermal MD treatments, although they also observed that the average atomic energy decreased during annealing. Hasnaoui et al. [14] also found that annealing computational nanocrystalline samples leads to a reduction in

\footnotetext{
*Corresponding author. Email: schuh@mit.edu
} 
the GB energy and a strengthening effect. In addition, Hasnaoui et al. observed that plastically deformed specimens exhibited relaxed GB structures which were qualitatively similar to annealed samples, although the effect of this mechanically driven relaxation on subsequent mechanical properties was not explored. The concept of interface modification driven by applied stress is common within the mechanochemistry community, where shear-driven mass transport is often observed as a result of plastic deformation [15-17].

In addition to being of scientific interest, the possibility that applied stresses can relax GBs in nanocrystalline materials may dramatically impact wear and fatigue properties. Mechanical relaxation strengthening could be advantageous in some such situations, where cyclic hardening could, e.g., retard wear processes, or on the other hand provide a local embrittlement mechanism that would promote cyclic fatigue crack advance. However, despite our prior speculations on this subject in the context of wear [5] and the observation that cyclic loading can encourage grain growth in nanocrystalline metals [5,18], we are not aware of any study that has explicitly established that local structural relaxation of GBs, with a concomitant strength increase, can occur due to cyclic loading in a nanocrystalline material. In this study, we do so, using atomistic simulations on nanocrystalline Ni.

\section{Simulation details}

MD simulations were performed using nanocrystalline $\mathrm{Ni}$ as a model system. An embedded atom method (EAM) potential which accurately reproduces mechanical properties as well as defect energies [19] was used, and all simulations were run with the LAMMPS code [20] using an integration time step of $1 \mathrm{fs}$. Nanocrystalline specimens with average grain sizes $d=3,4,5$, and $10 \mathrm{~nm}$ were created using a Voronoi tessellation construction modified to enforce a minimum separation distance ( $\sim 25 \%$ of the simulation cell length) between grain nucleation sites, giving more equiaxed grains and a tighter grain size distribution. The specimens with $d=3,4$, and $5 \mathrm{~nm}$ contained 24 grains and were geometrically similar (relative grain centers and orientation angles were kept constant for all three samples), while the $d=10 \mathrm{~nm}$ specimen contained 12 grains. All simulations employed periodic boundary conditions and a Nose-Hoover thermo/barostat. The specimens were initially equilibrated at $300 \mathrm{~K}$ and zero pressure until a steady-state system energy was reached $(500 \mathrm{ps}$ for the specimens with $d=3,4$, and $5 \mathrm{~nm}$, and $20 \mathrm{ps}$ for the $d=10 \mathrm{~nm}$ specimen). This method of initial equilibration is accepted within the computational materials science community for the creation of realistic nanocrystalline structures, and has been shown to reproduce experimentally measured values of sample density, GB density, and excess GB enthalpy [21,22]. These computational samples recreate the metastable equilibrium configuration of real nanocrystalline specimens in their as-deposited state, and have been widely used to validate and inform experimental studies of nanocrystalline mechanical behavior [23-27]. Deformation was simulated with uniaxial tension tests at an engineering strain rate of $5 \times 10^{8} \mathrm{~s}^{-1}$ while keeping zero stress on the other axes. Tension tests were performed in each orthogonal direction in order to average out any orientation 
dependence of mechanical properties and strength was measured by taking the $1 \%$ offset yield stress following Ref. [28].

A range of mechanical treatments were applied to the as-prepared computational samples. The first type of treatment involved the application of fully reversed cyclic uniaxial loading to a given maximum strain. These straining cycles were applied to each axial direction in sequence, so that all three axes underwent identical treatments. In some cases, the uniaxial treatments were applied multiple times. A second type of treatment involved the application of fully reversed hydrostatic expansions. A limited number of thermal annealing treatments (100 or $300 \mathrm{ps}$ at $600 \mathrm{~K}$, then cooled at $30 \mathrm{~K} / \mathrm{ps}$ ) were also carried out on specimens with $d=3 \mathrm{~nm}$, for comparison with the mechanical cycling simulations. GB atoms were distinguished from those in the grain interior using the centrosymmetry parameter (CSP) [29], using the Lindemann-Gilvarry rule [30], which suggests that, for $\mathrm{Ni}$, a CSP value $\geq 2.14 \AA^{2}$ is a defect (and can be classified as a GB atom in this case since we do not observe any stored dislocation or stacking fault networks within the grains).

\section{Results and discussion}

The yield stresses of the as-prepared and treated samples are shown as a function of GB fraction in Figure 1a and as a function of the average atomic energy in Figure 1b. In the case of the as-prepared specimens, yield stress scales apparently linearly with both the fraction of GB atoms and the average atomic energy of the system. The strength of these as-prepared samples decreases as grain size is decreased, in line with extensive prior literature [24,31]. The data for thermally treated structures are also in line with prior studies of thermally induced boundary relaxation $[12,13]$. We are not aware of prior work revealing the major trend in Figure 1, namely, that cyclic mechanical loading also increases the yield strength of the nanocrystalline samples. We do not observe that strengthening is always accompanied by a significant change in the fraction of GB atoms (Figure 1a), but the system energy is always reduced (Figure 1b), which speaks to the equilibration of the boundary structure; this is similar for both mechanical and thermal treatments (Figure 1b). In fact, the observed strengthening scales remarkably well with the average atomic energy of the system, but not with GB fraction; this suggests that the average atomic energy is a more nuanced measurement of the GB structural state, or at least those GB structural features that affect mechanical strength.

Figure 1c presents a magnified view of the yield stresses of the mechanically treated $d=3 \mathrm{~nm}$ specimens as a function of the average atomic energy, with the details of the mechanical treatments included in order to compare their efficacies in relaxing GB structure. As might be expected, the most effective mechanical treatments are those used in the case of larger strains and with multiple loading cycles, both of which favor more microplastic activity that can restructure GBs. Somewhat less intuitive is the result that hydrostatic expansions do not seem to cause significant GB relaxation, even when large dilatations (up to 4\%) are applied. These trends can be understood by inspecting the stress-strain behavior during the mechanical treatments, as shown in Figure 1d. For cycling either to $1 \%$ tensile strain or $3 \%$ hydrostatic strain, the loading and unloading curves overlap, there is no 

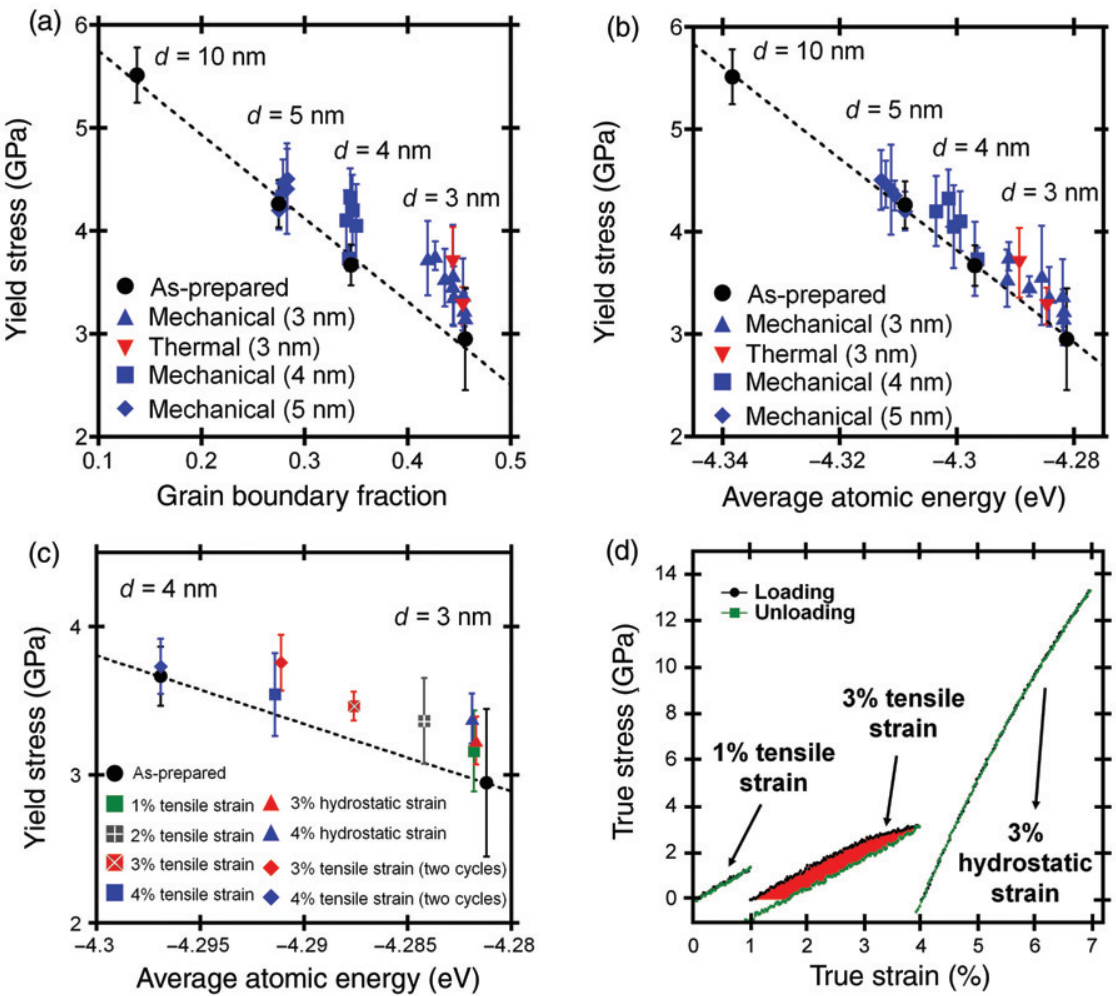

Figure 1. The yield stresses of as-prepared and treated specimens plotted as a function of: (a) GB fraction and (b) average atomic energy. While the strengths of the as-prepared samples scale with both quantities, the mechanically treated samples only scale well with the average atomic energy of the system. (c) A magnified view of the data for mechanically treated $d=3 \mathrm{~nm}$ samples shows that tensile treatments to larger strains and multiple loading cycles are more effective for relaxation strengthening. (d) Effective treatments cause dissipation of energy through plastic deformation, as shown by the stress-strain behavior along the $x$-axis.

permanent deformation, and the treatments do not cause strengthening. In both cases, this is apparently because no plastic deformation was triggered, i.e., no permanent atomic rearrangements occurred. However, cycling to $3 \%$ tensile strain induces some amount of plastic deformation that effectively relaxes the GBs and strengthens the system against further deformation.

Based on these observations, it appears that energy dissipation through plastic deformation is necessary for mechanical relaxation of GBs. While this seems similar to traditional dislocation-based cyclic hardening processes at first glance, it is important to point out that no stored crystal dislocation networks are introduced during the deformation of these nanocrystalline samples, making this a fundamentally different type of strengthening relegated to the GBs. Figure 2 compiles the change in system energy for the treated samples of all grain sizes as a function of the amount of energy dissipated during plastic deformation. All the data points lie 


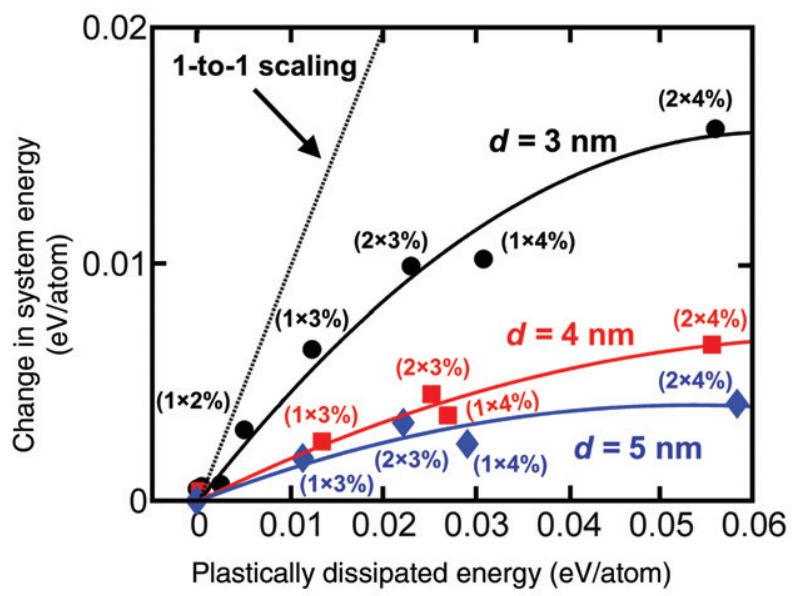

Figure 2. The change in the system energy plotted as a function of the energy dissipated during mechanical cycling. Each data point is labeled $(A \times B \%)$ to denote the mechanical treatment that was applied, where " $\mathrm{A}$ " represents the number of applied deformation cycles and " $\mathrm{B}$ " the applied tensile strain (the hydrostatic treatments lie very close to zero on this graph and were not labeled). A given level of GB relaxation requires less energy dissipation as grain size is decreased.

beneath the 1-to-1 line that represents equality, demonstrating that only a fraction of the dissipated energy goes toward relaxing the system energy. Moreover, the data show a trend that is concave down, suggesting that it becomes more difficult to evolve the system energy as relaxation progresses. In other words, more energy dissipation (i.e., more plastic deformation) is required to reduce the system energy by a given amount in a partially relaxed specimen than in an as-prepared specimen. This suggests that the mechanical strengthening effect saturates and becomes less efficient as excess GB defects are removed. In addition, more energy must be dissipated to cause a given change in system energy as grain size increases, suggesting that plastic deformation more efficiently relaxes GB structure at finer grain sizes. Such a trend persists even if the different data sets are normalized by GB area or number of GB atoms. In other words, this is not simply a geometrical effect due to an increasing GB volume with decreasing grain size, and samples with smaller grain size are apparently able to relax more for some physical reason associated with details of their deformation. This is reasonable, since as we will see later, the smaller grain sizes favor GB sliding and grain rotation, which are collective processes that could be expected to be more effective at relaxing non-equilibrium GB structures.

Atomistic details of the relaxation process can be found by inspecting the computational samples before and after selected mechanical treatments. The AtomEye program [32] was used to visualize the atomic configurations, with atoms colored according to their CSP values. Figure $3 \mathrm{a}$ and $\mathrm{b}$ show a $d=3 \mathrm{~nm}$ specimen in the as-prepared state and after two cycles to $4 \%$ tensile strain, respectively. Select crystal planes from the as-prepared state are marked with dashed white lines in both figures while solid white lines mark crystal planes from the mechanically treated state in Figure $3 b$, highlighting the fact that grain rotation has 
(a) As-prepared

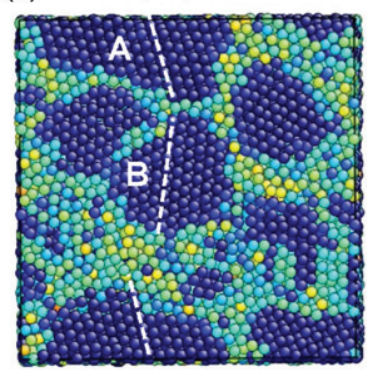

(b) Two cycles to $4 \%$ tensile strain

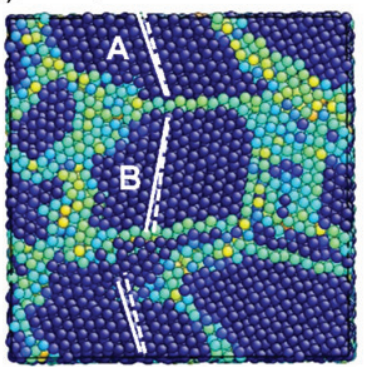

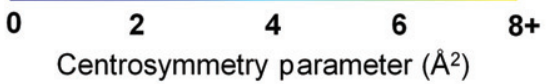

Figure 3. The $d=3 \mathrm{~nm}$ sample shown in (a) its as-prepared state and (b) after two cycles to $4 \%$ tensile strain. A reduction in the number of GB atoms is observed in the mechanically treated case, as well as the formation of low-energy boundaries. The dashed white fiducial markers are aligned with crystal planes in the as-prepared configuration presented in (a) and have the same orientation in (b). Similar planes in the mechanically treated configuration are denoted by solid white lines, showing the rotation of the grains.

taken place in the mechanically treated sample. The misorientation between the two grains labeled A and B is about $52^{\circ}$ on [13 129] in the as-prepared state, while it is $60^{\circ}$ about $\left[\begin{array}{lll}1 & 1 & 1\end{array}\right]$ in the mechanically cycled state; the misorientation between the grains changed by about $12^{\circ}$ as a result of mechanical cycling. This rotation has reduced the number of GB atoms observed in this image, and has in fact rendered the boundary coherent, i.e., a (1 111$) \Sigma 3$ twin boundary. This is one particularly clear example of how the system ratchets to a set of boundaries with lower energies and fewer local stress concentrations for the preferential initiation of plastic deformation. In the case of the $d=3 \mathrm{~nm}$ sample presented in Figure 3, a net decrease in the number of GB atoms and an alteration of GB character to low-energy configurations occur during mechanical cycling, both of which contribute to a reduction of the total system energy.

The obvious structural changes shown above for the $d=3 \mathrm{~nm}$ specimens are not observed in the cycled samples with larger grain sizes, which are more highly constrained due to their lower GB volume fractions. However, GB structure is observed to relax in more subtle ways. Figure $4 \mathrm{a}$ and $\mathrm{b}$ show the distributions of atomic energies for a $d=4 \mathrm{~nm}$ sample in the as-prepared state and after two cycles to $4 \%$ tensile strain, respectively. The average energy of GB atoms, represented by the dotted blue lines in Figure 4, is significantly reduced in the cycled sample when compared to the as-prepared state. Although the number of GB atoms remains the same in this case (recall Figure 1a), high-energy GB sites are relaxed to lower energy configurations, leading to an overall drop in the system energy (the dashed black line in Figure 4). Figure 4 also demonstrates why the average atomic energy of the system provides a better measurement of GB relaxation than the GB fraction does; the average atomic energy incorporates information about the GB fraction, since these 

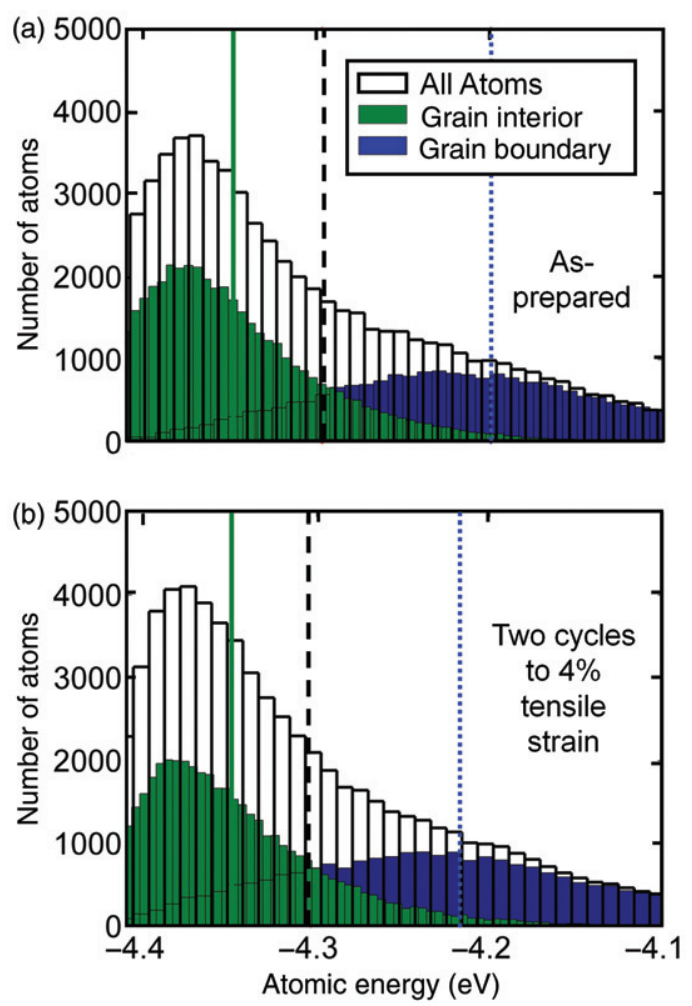

Figure 4. The atomic energy distributions for a $d=4 \mathrm{~nm}$ sample in (a) the as-prepared state and (b) after two cycles to 4\% tensile strain. Although the GB fraction remains constant after mechanical cycling, the energetic state of the GB atoms is greatly reduced, which also results in a reduction of the overall system energy.

atoms generally have higher atomic energies than grain interior atoms, as well as information about the energetic states of the GB atoms.

\section{Conclusions}

This study shows that the GB state and strength of nanocrystalline metals can be modified by the application of mechanical treatments. These results should be of interest for studying fatigue and wear properties, as a strengthening effect is predicted under cyclic mechanical loading, with the strength increments measured here being as large as $\sim 27 \%$. These results also align with observations from the experimental literature. Our own prior work showed that nanocrystalline Ni-W alloys harden under repetitive sliding loads, leading to unexpected improvements in wear resistance in alloys with extremely fine grain sizes [5]. The largest wear-induced hardening effect occurred at the finest grain sizes (as fine as $d=3 \mathrm{~nm}$ ), which agrees with the present results. Moser et al. [33] observed a cyclic hardening effect in 
electrodeposited nanocrystalline $\mathrm{Ni}$ with $d=40 \mathrm{~nm}$ subjected to cyclic tensiontension deformation, and rationalized that this behavior was the result of the exhaustion of GB dislocation sources. Although our results are more applicable at finer grain sizes where only GB deformation occurs, they agree with the general mechanistic explanation of Moser et al. in the sense that they reveal that small amounts of plastic deformation can relax GBs and render them mechanically stronger. Low-energy configurations are found during mechanical cycling, either through an alteration in GB character or subtle rearrangement within the GB, thereby reducing local stress concentrations at the boundary and making further plastic deformation more difficult.

\section{Acknowledgements}

This study was supported by the US Army Research Office, through grant W911NF-09-10422 and through the Institute for Soldier Nanotechnologies at MIT.

\section{References}

[1] M. Dao, L. Lu, R.J. Asaro, J.T.M. De Hosson and E. Ma, Acta Mater. 55 (2007) p.4041.

[2] J.R. Weertman, D. Farkas, K. Hemker, H. Kung, M. Mayo, R. Mitra and H. Van Swygenhoven, MRS Bulletin 24 (1999) p.44.

[3] Z.N. Farhat, Y. Ding, D.O. Northwood and A.T. Alpas, Mater. Sci. Eng., A 206 (1996) p.302.

[4] D.H. Jeong, F. Gonzalez, G. Palumbo, K.T. Aust and U. Erb, Scr. Mater. 44 (2001) p.493.

[5] T.J. Rupert and C.A. Schuh, Acta Mater. 58 (2010) p.4137.

[6] T. Hanlon, E.D. Tabachnikova and S. Suresh, Int. J. Fatigue 27 (2005) p.1147.

[7] H.A. Padilla and B.L. Boyce, Exp. Mech. 50 (2010) p.5.

[8] K.S. Kumar, H. Van Swygenhoven and S. Suresh, Acta Mater. 51 (2003) p.5743.

[9] D. Jang and M. Atzmon, J. Appl. Phys. 99 (2006) p.083504.

[10] Y.M. Wang, S. Cheng, Q.M. Wei, E. Ma, T.G. Nieh and A. Hamza, Scr. Mater. 51 (2004) p.1023.

[11] A.J. Detor and C.A. Schuh, J. Mater. Res. 22 (2007) p.3233.

[12] N.Q. Vo, R.S. Averback, P. Bellon and A. Caro, Phys. Rev. B 78 (2008) p.241402.

[13] N.Q. Vo, R.S. Averback, P. Bellon and A. Caro, Scr. Mater. 61 (2009) p.76.

[14] A. Hasnaoui, H. Van Swygenhoven and P.M. Derlet, Acta Mater. 50 (2002) p.3927.

[15] F. Delogu, J. Phys. Condens. Matter 19 (2007) p.096008.

[16] F. Delogu and G. Cocco, Phys. Rev. B 71 (2005) p.144108.

[17] V.I. Levitas, Phys. Rev. B 70 (2004) p.184118.

[18] J. Schiotz, Mater. Sci. Eng. A 375 (2004) p.975.

[19] Y. Mishin, D. Farkas, M.J. Mehl and D.A. Papaconstantopoulos, Phys. Rev. B 59 (1999) p.3393.

[20] S. Plimpton, J. Comput. Phys. 117 (1995) p.1.

[21] H. Van Swygenhoven, D. Farkas and A. Caro, Phys. Rev. B 62 (2000) p.831.

[22] H. Van Swygenhoven, M. Spaczer and A. Caro, Nanostruct. Mater. 12 (1999) p.629.

[23] S. Jang, Y. Purohit, D.L. Irving, C. Padgett, D. Brenner and R.O. Scattergood, Acta Mater. 56 (2008) p.4750.

[24] J. Schiotz and K.W. Jacobsen, Science 301 (2003) p.1357. 
[25] J. Schiotz, T. Vegge, F.D. Di Tolla and K.W. Jacobsen, Phys. Rev. B 60 (1999) p.11971.

[26] H. Van Swygenhoven, P.M. Derlet and A.G. Froseth, Acta Mater. 54 (2006) p.1975.

[27] N.Q. Vo, R.S. Averback, P. Bellon, S. Odunuga and A. Caro, Phys. Rev. B 77 (2008) p.134108.

[28] S. Brandstetter, H. Van Swygenhoven, S. Van Petegem, B. Schmitt, R. Maass and P.M. Derlet, Adv. Mater. 18 (2006) p.1545.

[29] C.L. Kelchner, S.J. Plimpton and J.C. Hamilton, Phys. Rev. B 58 (1998) p.11085.

[30] J.J. Gilvarry, Phys. Rev. 102 (1956) p.308.

[31] J. Schiotz, F.D. Di Tolla and K.W. Jacobsen, Nature 391 (1998) p.561.

[32] J. Li, Modell. Simul. Mater. Sci. Eng. 11 (2003) p.173.

[33] B. Moser, T. Hanlon, K.S. Kumar and S. Suresh, Scr. Mater. 54 (2006) p.1151. 